\title{
Direct digital-to-RF converter employing semi- digital FIR voltage-mode RF DAC
}

Mohammad Reza Sadeghifar, Hakan Bengtsson, Jacob Wikner and Oscar Gustafsson

The self-archived postprint version of this journal article is available at Linköping University Institutional Repository (DiVA):

http://urn.kb.se/resolve?urn=urn:nbn:se:liu:diva-158579

N.B.: When citing this work, cite the original publication.

Sadeghifar, M. R., Bengtsson, H., Wikner, J., Gustafsson, O., (2019), Direct digital-to-RF converter employing semi-digital FIR voltage-mode RF DAC, Integration, 66, 128-134.

https://doi.org/10.1016/j.vlsi.2019.02.005

Original publication available at:

https://doi.org/10.1016/j.vlsi.2019.02.005

Copyright: Elsevier

http://www.elsevier.com/

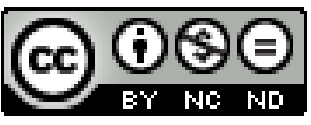




\title{
Direct Digital-to-RF Converter Employing Semi-Digital FIR Voltage-Mode RF DAC
}

\author{
M Reza Sadeghifar ${ }^{\mathrm{a}, \mathrm{b}, *}$, Håkan Bengtsson ${ }^{\mathrm{b}}$, J Jacob Wikner ${ }^{\mathrm{a}}$, Oscar \\ Gustafsson $^{\mathrm{a}}$ \\ ${ }^{a}$ Department of Electrical Engineering, Linköping University, SE-581 83 Linköping, Sweden \\ ${ }^{b}$ Ericsson AB, Stockholm, Sweden
}

\begin{abstract}
A direct digital-to-RF converter (DRFC) is presented in this work. Due to its digital-in-nature design, the DRFC benefits from technology scaling and can be monolithically integrated into advance digital VLSI systems. A fourthorder single-bit quantizer bandpass digital $\Sigma \Delta$ modulator is used preceding the DRFC, resulting in a high in-band signal-to-noise ratio (SNR). The out-ofband spectrally-shaped quantization noise is attenuated by an embedded semidigital FIR filter (SDFIR). The RF output frequencies are synthesized by a novel configurable voltage-mode RF DAC solution with a high linearity performance. The configurable RF DAC is directly synthesizing RF signals up to $10 \mathrm{GHz}$ in first or second Nyquist zone. The proposed DRFC is designed in $22 \mathrm{~nm}$ FDSOI CMOS process and with the aid of Monte-Carlo simulation, shows $78.6 \mathrm{dBc}$ and $63.2 \mathrm{dBc}$ worse case third intermodulation distortion (IM3) under process mismatch in $2.5 \mathrm{GHz}$ and $7.5 \mathrm{GHz}$ output frequency respectively.

Keywords: Direct digital-to-RF Converter; DRFC; Semi-Digital FIR; RF

DAC; Digital Sigma Delta
\end{abstract}

Declarations of interest: none

\footnotetext{
${ }^{*}$ Corresponding author

Email addresses: reza.sadeghifar@ericsson.com (M Reza Sadeghifar), hakan.bengtsson@ericsson.com (Håkan Bengtsson), jacob.wikner@liu.se (J Jacob Wikner), oscar.gustafsson@liu.se (Oscar Gustafsson)
}

Preprint submitted to Elsevier

February 25, 2019 


\section{Introduction}

Today's wireless communication systems demand very high data capacity while the size and power consumption requirement of the hardware equipment decreases continuously. This will be achievable by hard integration of the dig-

5 ital circuitry and the RF frond-end into a single true VLSI system-on-chip solution [1. The analog and RF front-end of a wireless communication system, traditionally use a different technology process than digital circuitry due to the voltage headroom and other limitations which makes the higher integration of wireless systems in the same die, more challenging. In this work we are proposing a novel digital-to-RF converter (DRFC) for digital IQ transmitter that is capable of monolithic integration into digital VLSI due to its digital-in-nature design. The digital transmitter, as shown in Fig. 1, features a IQ digital modulator, a bandpass $\Sigma \Delta$ modulator and a DRFC with embedded SDFIR and weighted one-bit RF DACs as SDFIR filter taps.

The rest of the paper is formatted as follows: In Section 2, digital IQ transmitter architecture is reviewed, in Section 3 a brief background on semi-digital FIR filter is given. The proposed DRFC is described in Section 4 and systemlevel simulation is presented in Section 4. Details of circuit implementation and circuit-level simulation analysis and impact of non-idealities are given in Section 6 and finally conclusion remarks come in Section 8 .

\section{Digital IQ transmitter Architecture}

Using a digital-to-RF converter (DRFC) has the advantage that by employing an RF DAC which directly synthesizes the RF frequencies, the need for analog frequency translation is removed and hence the transmitter chain can be more compact. In fact the complexity of the transmitter is pushed towards digital which benefits from implementation point of view, such as power and cost perspective. The latest trend also shows that digital-intensive transceiver is the way forward to address the higher capacity and emerging wireless technologies [2, 3, 4, 5]. Figure 1 shows the block diagram of a digital IQ transmitter 


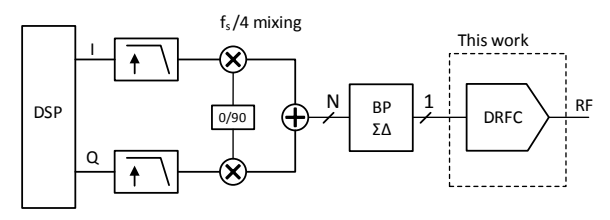

Figure 1: Block diagram illustration of a digital IQ transmitter employing a BP $\Sigma \Delta$ Modulator and a DRFC.

30 featuring a DRFC. The IQ modulator is followed by a $\Sigma \Delta$ noise-shaper and a direct digital-to-RF converter (DRFC) [6, 7, 8, 9]. The I and $\mathrm{Q}$ data are run at baseband sample rate. A digital up-converter performs the IQ modulation and locate the baseband signal in desired IF frequency. The up-converted digital data can be directly applied to a high resolution RFDAC or can be implemented

35 as BP $\Sigma \Delta$ and semi-digital FIR filter [10, 11, 12]. The choice of architecture, however, depends on the trade-off between in-band resolution and out-of-band emission [13. A $\Sigma \Delta$ modulator followed by a semi-digital FIR filter as a DRFC is beneficial in WLAN applications where a high in-band resolution digital-toanalog conversion that meets the out-of-band spectrum emission mask can be

40 efficiently implemented [12]. In this work, a novel semi-digital RF DAC implementation is presented.

\subsection{Digital IQ modulator with $f_{s} / 4$}

The digital IQ modulation can be greatly simplified if the IF frequency is located at $f_{s} / 4$. The digital oscillating signal will then be simple stream of 451,0 and -1 values and the multiplication can be efficiently implemented in hardware [10]. If the IF frequency is not at $f_{s} / 4$, the digital IQ modulator should utilize a numerically controlled oscillator (NCO) to frequency shift the input signal to the desired IF frequency, which requires more hardware resources and has higher power consumption.

\subsection{Bandpass Digital Sigma Delta Modulator}

A bandpass $\Sigma \Delta$ modulator can be utilized to spectrally shape the quantization noise to the output of band of interest. Therefore the same high resolution 
signal can be represented after $\Sigma \Delta$ by much fewer bits. This simplifies the succeeding digital-analog conversion stage. However the quantization noise shaped to the out of band, in most cases needs to be attenuated to meet the wireless standard's spectral emission mask. The $\Sigma \Delta$ can be also a bandpass modulator with tunable notch frequency [14].

\section{Semi-Digital FIR filter}

Semi-digital FIR filters or sometimes called analog FIR filters, are used as frequency selective filters as well as converting from digital domain to analog domain since it uses analog multipliers as filter taps. The analog multipliers are implemented by means of current sources, in conventional SDFIR filters, if the output of the $\Sigma \Delta$ modulator quantizer is a single bit [15, 16, 17, 18, 19, 20, 21, 12, 22. In fact each tap is one-bit DAC and they are weighted according to the filter coefficients. The single bit stream is traveling through the FIR delay elements and multiplied by analog taps. Each taps output is in current mode and the overall SDFIR output is simply the combination of currents which is terminated in a load impedance. Although most reported SDFIR filters are one bit, in transmitter in [23, a multi bit SDFIR with very few taps is utilized to create a frequency notch at desired receiver band.

\subsection{FIR Filter Coefficients Precision}

The SDFIR filter design procedure is normally similar to that of digital FIR filters. However there are two considerations. Firstly, since the SDFIR filter taps are implemented in analog current sources, the precision of the filter coefficients cannot be too large. In this work however, voltage-mode analog multipliers are proposed as shown in Fig. 2. Quantization error introduced by the limited coefficient precision will alter the FIR filter frequency response [15, 17, 19, 21, 12. Secondly the SDFIR, in contrary to digital FIR, will have mismatch between the multipliers and this inaccuracy in FIR filter coefficients imposes a higher bound on achievable attenuation by the SDFIR filter [24, 25]. A 
compromise needs to be reached between coefficient limited precision error and mismatch error. Therefore very high coefficient precision will be unnecessary as the mismatch among analog multipliers will destroy the excessive attenuation gained by higher precision.

In this work, the filter is designed for a linear-phase response. The coefficient mismatch will lead to the filter slightly deviating from the linear-phase requirement. One may argue that this combined with the longer filter (more filter taps) required for linear-phase filters make a non-linear-phase filter a better option. However, this must be evaluated at a system level as using a linear-phase filter will put less requirements on the equalizer at the receiver side, so from a receiver perspective a linear-phase design will be preferred.

\section{Proposed DRFC Architecture}

A top-level functional block diagram of the proposed DRFC for single-bit $\Sigma \Delta$ modulator, is shown in Fig. 2. The output bit from each delay element (DFF) and its complement bit are passed to the differential one-bit RFDAC. The one-bit RF DAC conversion cells are weighted according to the SDFIR filter coefficients. The negative numbers in coefficients are implemented by swapping the polarity of differential output in RF DAC conversion cells as exemplified in Fig. 2 for the second tap $h_{1}<0$.

Whether the normal mode or mixing mode of the RF DAC is selected, the bits are bypassed or XNOR-gated with the clock signal, respectively. Signal M selects the operation mode of the RF DAC. The inverter inside the RF DAC block acts as a switch and connects the unit element resistor $\left(R_{u}\right)$ to positive or negative reference voltage based on the input bit. The positive and negative output of all conversion cells are combined and terminated in a differential $100 \Omega$ load.

In the proposed DRFC solution, IQ modulation with $f_{s} / 4$ and bandpass $\Sigma \Delta$ modulator with notch frequency of $f_{s} / 4$ is considered and the SDFIR filter is designed for pass band at $f_{s} / 4$. In general with tunable bandpass $\Sigma \Delta$ modu- 


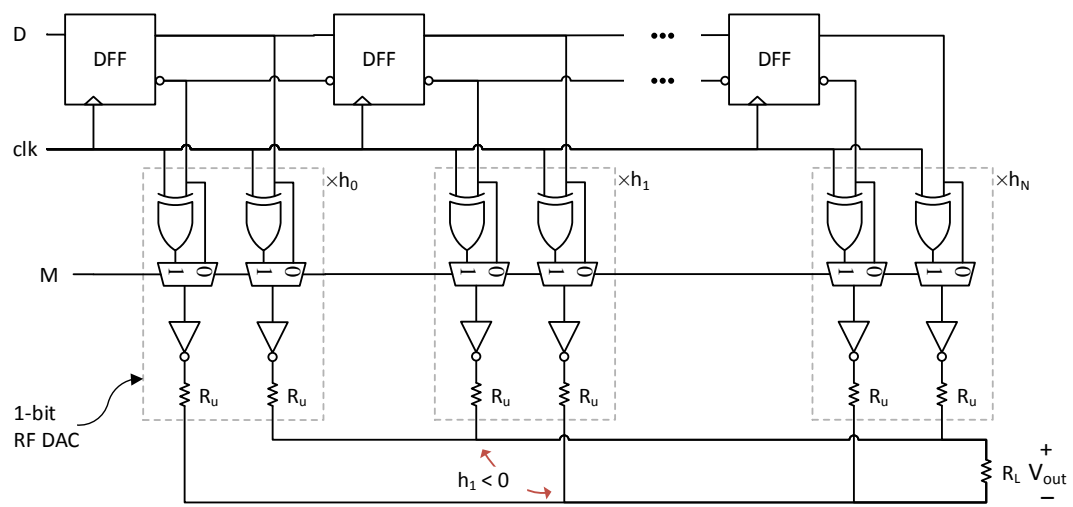

Figure 2: Functional block diagram illustration of direct digital-to-RF converter employing SDFIR and one-bit voltage-mode differential RFDAC.

lator, employing NCO in IQ modulator, and designing bandpass SDFIR filter accordingly, the proposed DRFC can cover other output frequencies than $f_{s} / 4$ as well. However to be able to have a tunable DRFC, the SDFIR needs to be programmable which is out of scope of this work. In this work the output frequency has a constant ratio with sample frequency and to sweep different output frequencies, the sample frequency can be changed. However, this requires resampler circuitry in sample rate generation as the baseband rate and sample rate will not have integer ratio.

\subsection{Voltage-Mode Mixing RF DAC}

RF DAC operation is subdivided into mixing logic and output stage in voltage-mode that will be discussed here.

\section{Mixing Logic}

Depending on the architecture and implementation choices, the RF DAC can utilize first, second or even higher Nyquist zones to synthesize the signal at the desired RF frequency. In a zero-order hold RF DAC, the analog signal is reconstructed by means of pulse-amplitude modulation (PAM) of the digital input data using a rectangular pulse with duration of sampling period $T_{s}=1 / f_{s}$, and the frequency response is a sinc-weighted function with zeros at multiples 


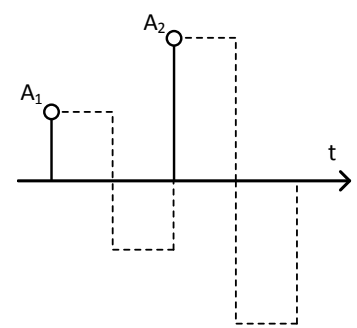

Figure 3: Bipolar pulse amplitude modulation (PAM) to generate the RF DAC frequency response.

of the sample frequency $f_{s}$. The sample rate in this case must be very high, for instance, more than $20 \mathrm{GHz}$ to cover up to $10 \mathrm{GHz}$ RF output within the first Nyquist zone. In mixing RF DACs, however, the PAM signal can be an oscillating pulse, for which the amplitude is modulated with the level of the input digital code. The frequency response of the RF DAC then will be a shifted sinc function, with a high energy lobe at $f_{s}$ or a multiple of $f_{s}$ depending on the oscillating pulse duration. The aliasing image of the digital data at $f_{s}$ (or a multiple of $f_{s}$ ), will be the RF output signal of the RF DAC [26, 6, 4, 27]. In general two approaches exist for implementing a mixing RF DAC depending on how the mixing operation in each cell of the DAC is performed, as discussed in [4]: at the data path as "mixing logic" or modulating the tail current as "series mixing" and therefore various oscillating PAM signals have been used for RF DAC implementation such as continues sinusoidal 26, 6, discrete oscillating PAM 28, or a bipolar rectangular [4, 27. In order to implement the RF DAC in a process that is compatible with digital ASIC, mixing logic approach is preferred due to the voltage headroom limitations and also the "digital-innature" characteristic of this method, so that the RF DAC actually benefits from process scaling. To implement the mixing logic function, the PAM signal is selected to be a bipolar pulse toggling between amplitudes $+A$ and $-A$, where $A$ is the level corresponding to the input digital code. The time-domain output signal will be as shown in Fig. 3. This PAM signal can be generated by applying an exclusive NOR logic gate (XNOR) to the clock and the input bit. This is 


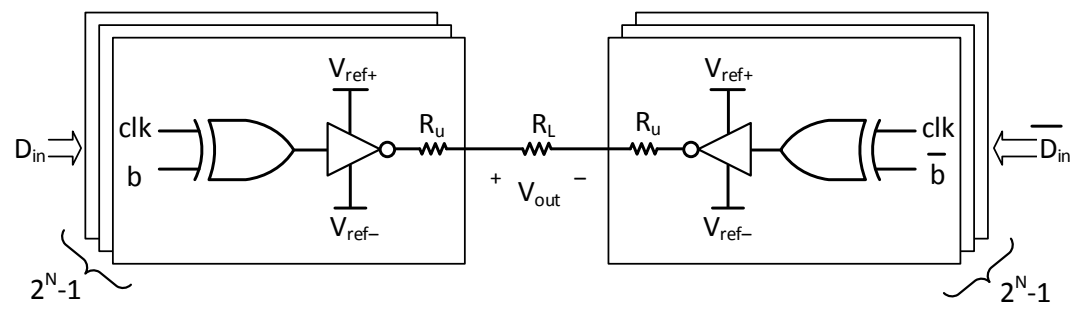

Figure 4: Mixing logic operation by XNOR-gating the input bit and the clock.

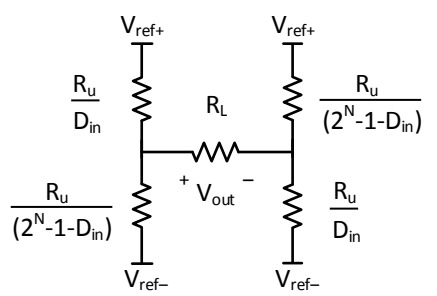

Figure 5: Simplified equivalent circuit at each clock phase.

150 are controlled by $\mathrm{N}$ bits.

\section{Output Stage of RF DAC in Voltage-Mode}

Traditionally current mode digital-to-analog conversion cells are utilized due to its high speed capability and not requiring an analog buffer at the output which is usually bandwidth limited. One of the main limitations of dynamic performance in current steering DACs, is the finite output impedance of the unit current cell, making the DAC output impedance input code-dependent, and causes non-linearity [29, 30]. Here we have proposed to use a voltage-mode RF DAC cell in SDFIR filter as shown in Fig. 4. At each clock phase the output stage will become a resistor network as shown in Fig. 5, and the output voltage can be calculated as a function of the input code. It is observable that for general $N$-bit case of voltage-mode RF DAC in Fig. 5 three KVL equations 
can be written as:

$$
\begin{aligned}
& \left(\frac{R_{u}}{K-D_{\text {in }}}+\frac{R_{u}}{D_{\text {in }}}+R_{L}\right) I_{1}+R_{L} I_{2}+\frac{R_{u}}{D_{\text {in }}} I=0 \\
& R_{L} I_{1}+\left(R_{L}+\frac{R_{u}}{K-D_{\text {in }}}+\frac{R_{u}}{D_{\text {in }}}\right) I_{2}-\frac{R_{u}}{K-D_{\text {in }}} I=0 \\
& \frac{R_{u}}{D_{\text {in }}} I_{1}-\frac{R_{u}}{K-D_{\text {in }}} I_{2}+\left(\frac{R_{u}}{K-D_{\text {in }}}+\frac{R_{u}}{D_{\text {in }}}\right) I=V,
\end{aligned}
$$

where $K=2^{N}$ - 1, i.e., $K$ represents the maximum of $D_{\text {in }}, R_{L}$ is the differential load, and $V=\left(V_{\text {ref+ }}-V_{\text {ref- }}\right) . I_{1}, I_{2}$, and $I$ are the currents in upper loop, lower loop and from supply voltage to the left branch. By solving the systems of equation above we have $I_{1}$, and $I_{2}$ as

$$
\begin{aligned}
& I_{1}=-\frac{\left(K-D_{\mathrm{in}}\right)\left(D_{\mathrm{in}} R_{L}+R_{u}\right)}{R_{u}\left(K R_{L}+2 R_{u}\right)} V \\
& I_{2}=-\frac{D_{i n}\left(D_{\mathrm{in}} R_{L}-K R_{L}-R_{u}\right)}{R_{u}\left(K R_{L}+2 R_{u}\right)} V,
\end{aligned}
$$

and hence the differential output voltage as

$$
\begin{aligned}
V_{\text {out }} & =R_{L}\left(I_{1}+I_{2}\right) \\
& =\frac{2 D_{\text {in }}-\left(2^{N}-1\right)}{\left(2^{N}-1\right)+2 R_{u} / R_{L}} V .
\end{aligned}
$$

This can also be achieved by investigating the half-differential Thevenin equivalent as:

$$
\begin{aligned}
V_{t h} & =\frac{D_{\text {in }}}{2^{N}-1} \cdot\left(V_{\text {ref }+}-V_{\text {ref- }}\right), \\
R_{t h} & =\frac{R_{u}}{2^{N}-1} .
\end{aligned}
$$

The differential output voltage is then derived as

$$
V_{\text {out }}=\frac{2 D_{\text {in }}-\left(2^{N}-1\right)}{\left(2^{N}-1\right)+2 R_{u} / R_{L}} \cdot\left(V_{\text {ref }+}-V_{\text {ref- }}\right) .
$$

In this RF DAC architecture, the impedance seen from the output, is always constant regardless of what input code is applied. That is, all the $R_{u}$ resistors are either connected to positive or negative reference voltage and hence the total output impedance is always constant and it does not contribute to the linearity degradation. As can be observed from (5), the output voltage is a linear function of the input code and there is no code-dependent load variation, as there was 
in current steering DACs. The "on" resistance of the switches is negligible Toolbox 31, has been used. A $4^{t h}$-order bandpass $\Sigma \Delta$ modulator with center frequency at $f_{s} / 4$, with one bit quantizer level is designed. A $78^{\text {th }}$-order bandpass SDFIR filter is also designed in MATLAB with Least-squares linear-phase FIR filter design function and the coefficients are rounded to 11-bit precision. at $3 f_{s} / 4$ instead. 

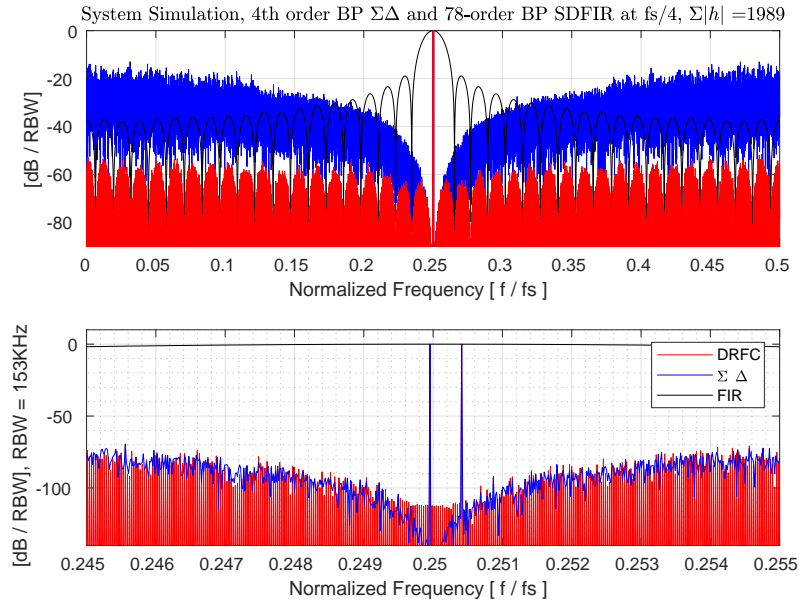

Figure 6: Behavioral-level simulation of the $\Sigma \Delta$ modulator, SDFIR filter response and the DRFC. The first Nyquist zone is illustrated. Wide-span spectrum is shown at the top and zoom-in span is shown at the bottom.

\section{RFDAC Conversion Cell Implementation}

The transistor-level schematic diagram of a unit conversion cell, consisting of data capturing latch, re-timing latch and mixing logic, and voltage-mode conversion stage, is shown in Fig. 7. The proposed DRFC solution is implemented in $22 \mathrm{~nm}$ fully depleted silicon-on-insulator (FDSOI) CMOS technology and simulated with the spectre simulator engine. Signal $b$ is the input bit to each cell using a differential clock signal $c l k_{p}$ and $c l k_{n}$ with 5 -ps rise- and fall times. Signal $M$ is selecting mixing or base-band mode operation of the RF DAC. $V_{\text {ref }}$ and $V_{\text {ref- }}$, are the reference voltages to the output stage to be provided by two band-gap reference and in this simulation, 0.8 and $0 \mathrm{~V}$ is used respectively.

\subsection{Impact of Mismatch}

Process and mismatch variation within RF DAC cells will cause errors in timing and amplitude accuracy in multi-bit RF DAC [32. But in one-bit RFDAC

there will be no internal mismatch variation. However coefficient inaccuracy of the SDFIR taps (one-bit RF DACs) results in SDFIR filter transfer function 


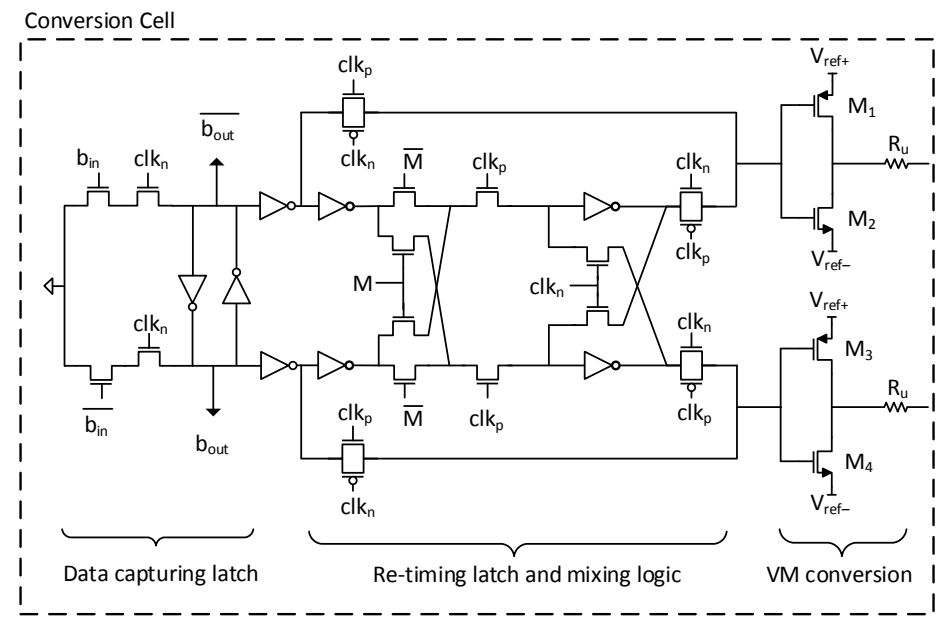

Figure 7: Conversion cell schematic.

variation [33, 24]. The variation in the passband magnitude can be neglected since in passband $|H(\omega)|=1$ and coefficients' error standard deviation is usually much smaller to make a noticeable error in the passband 24]. However in the stopband the mismatch effect is more significant and the reason is that in stopband the desired magnitude is very small and any deviation of the coefficients value from the ideal FIR taps will cause deviation from the desired frequency response in stopband. Actually there will be an upper bound for the expected attenuation in the stopband frequency range as derived in [24]. It should be noted that in transversal filters in [24] and [33], the assumption is that, a coefficient error can be modeled as an additive random variable whose variance does not depend on the desired coefficient value. However in SDFIR implementation with Poly resistors as proposed here or even with current sources as in [15, 17, the additive random variable's standard deviation actually depends on the desired coefficient value. According to Pelgrom's model [34, 35, the standard deviation of mismatch is inversely proportional to the area, and different coefficients have different device area. In order to characterize the resistor mismatch behavior in the selected process, $22 \mathrm{~nm}$ FDSOI CMOS, a single nominal $10 \mathrm{k} \Omega$ Poly resistor with size of $\mathrm{W}=0.36 \mu \mathrm{m}$ and $\mathrm{L}=7.6 \mu \mathrm{m}$ and $h_{k}$ resistors of 
$10 \mathrm{k} \Omega$ in parallel $\left(h_{k}\right.$ is FIR coefficient and as an example $h_{k}=64$ ), is simulated with 1000 Monte-Carlo samples and the simulated resistance value is observed. The simulation is run in three different mode, first only mismatch, second only

\begin{tabular}{l|lll} 
& Mismatch & Process & $\begin{array}{l}\text { Mismatch } \\
\text { and process }\end{array}$ \\
\hline$\epsilon, \Omega$ & 124.1 & 699.1 & 665 \\
$\mu, \Omega$ & $10.01 \mathrm{k}$ & $10.03 \mathrm{k}$ & $10.03 \mathrm{k}$ \\
$\sigma, \%$ & $1.24 \%$ & $6.97 \%$ & $6.6 \%$
\end{tabular}

Table 1: One unit element resistor $R_{u}$ with nominal value of $10 \mathrm{k} \Omega$.

process and third with both mismatch and process variation and the one-sigma standard deviation values and mean values are listed in Table 1 and 2 , where $225 \epsilon$ is a random error that represents the fluctuation around the nominal value of the resistor in $\Omega$, and $\mu$ is mean value in $\Omega$ and $\sigma$ is standard deviation of the resistor in percentage relative to the mean value. As expected from Pelgrom's model [34, 35, the mismatch variation (within die) is scaled with the square root of area, i.e., 64 parallel resistors have 64 times more area and hence the corresponding sigma is 8 times smaller $(0.16 \%=1.24 \% / \sqrt{64})$, while the process variation (die-to-die) does not scale with area. Although for the dynamic performance it is the mismatch variation only which is important, to ensure that it covers the worse cases, the simulations in the following sections are run with both mismatch and process variation indicating that the simulation result is conservative.

\begin{tabular}{l|lll} 
& Mismatch & Process & $\begin{array}{l}\text { Mismatch } \\
\text { and process }\end{array}$ \\
\hline$\epsilon, \Omega$ & 0.249 & 10.92 & 10.38 \\
$\mu, \Omega$ & 156.3 & 156.7 & 156.7 \\
$\sigma, \%$ & $0.16 \%$ & $6.97 \%$ & $6.6 \%$
\end{tabular}

Table 2: 64 unit element resistors $R_{u}$ in parallel (example $h_{k}=64$ ) with nominal value of $10 k / 64=156 \Omega$. 
There are $\sum|h|$ resistors in parallel at each clock phase, connected to the reference voltages. The uncorrelated noise power is added from each resistor

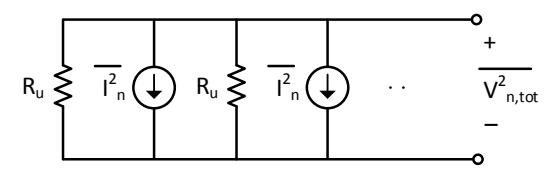

Figure 8: Equivalent noise circuit.

and the total noise power will be

$$
\begin{gathered}
\overline{I_{n, \text { tot }}^{2}}=\overline{I_{n, 1}^{2}}+\overline{I_{n, 2}^{2}}+\ldots=\frac{4 k T}{\left(\frac{R_{u}}{\sum|h|}\right)}, \\
\overline{V_{n, \mathrm{tot}}^{2}}=\overline{I_{n, \mathrm{tot}}^{2}}\left(\frac{R_{u}}{\sum|h|}\right)^{2}=4 k T\left(\frac{R_{u}}{\sum|h|}\right),
\end{gathered}
$$

which implies that the thermal noise power of all resistors in parallel, is divided by the total number of unit elements.

\section{Circuit-level Simulation}

The circuit-level simulation results confirm the theoretical discussion on linearity performance in the previous sections. The bandpass $\Sigma \Delta$ modulator is simulated in MATLAB and the $\Sigma \Delta$-modulated signal is imported to CADENCE design environment. The DRFC circuit designed in $22 \mathrm{~nm}$ FDSOI CMOS is simulated with this $\Sigma \Delta$-modulated test signal with Spectre simulator engine. For RF signals within first Nyquist zone, the normal mode of operation of the RF DAC is selected and for RF signals at second Nyquist zone, the RF DAC is configured to operate in mixing mode. A two tone test is performed on the circuit-level implementation. Differential load of $100 \Omega$, with $300 \mathrm{fF}$ capacitive load at each side, is used. Third order intermodulation distortion (IM3) is measured by this two-tone test, each tone at $-6 \mathrm{dBFS}$, and $7 \mathrm{MHz}$ frequency spacing 

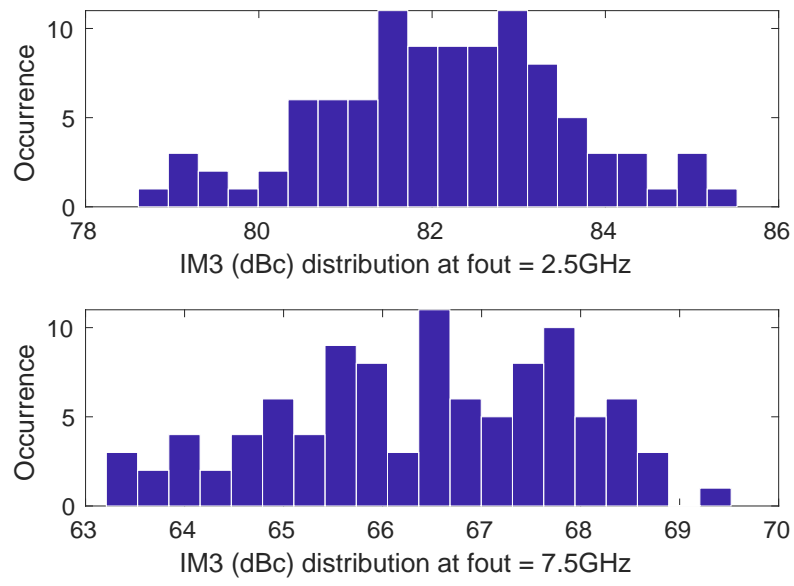

Figure 9: Distribution of 100-point Monte-Carlo IM3 (dBc) simulation for two output frequencies of $2.5 \mathrm{GHz}$ and $7.5 \mathrm{GHz}$.

between tones. The DRFC is sampling at 10 Gsps and the output frequency is at $f_{s} / 4=2.5 \mathrm{GHz}$ for normal mode operation of DRFC and at $3 f_{s} / 4=7.5 \mathrm{GHz}$ for mixing mode operation, respectively.

In order to analyze the unit element resistors mismatch impact, 100-point Monte-Carlo simulation is run and Fast Fourier transform (FFT) with $2^{18}$ points bution of IM3 results for output frequencies of $2.5 \mathrm{GHz}$ and $7.5 \mathrm{GHz}$ is shown in Fig. 9. The average and worse-case IM3 performance at $f_{\text {out }}=2.5 \mathrm{GHz}$ is $82.1 \mathrm{dBc}$ and $78.6 \mathrm{dBc}$ respectively. At $f_{\text {out }}=7.5 \mathrm{GHz}$, the average and worse-case IM3 performance is $66.4 \mathrm{dBc}$ and $63.2 \mathrm{dBc}$ respectively.

The output spectrum is demonstrated in Fig. 10 for one of the cases of Monte-Carlo simulation for two output frequencies of $2.5 \mathrm{GHz}$ and $7.5 \mathrm{GHz}$. On the left side the IM3 measurement is shown and on the right side the far-out spectrum is shown within full Nyquist bandwidth.

In order to compare the performance results of the proposed DRFC with the state-of-art, few works are listed here. In [36], a current steering SDFIR DAC with FIR filter order of 63 is reported. Due to the challenges in analog part, 


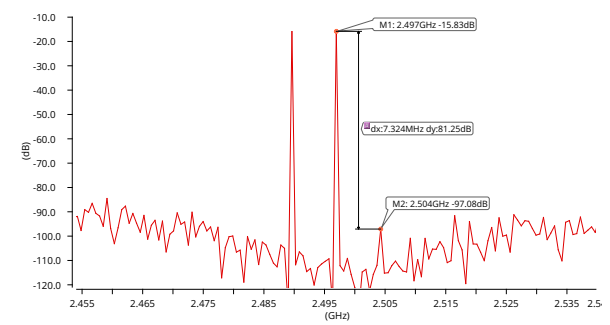

(a)

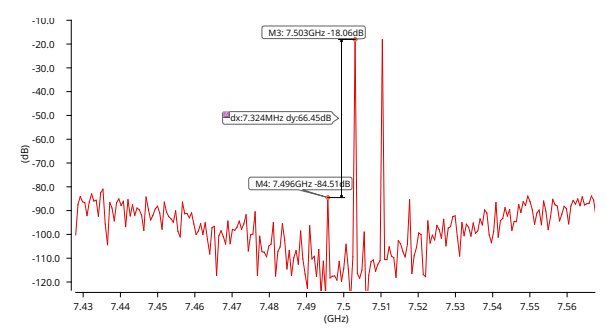

(c)

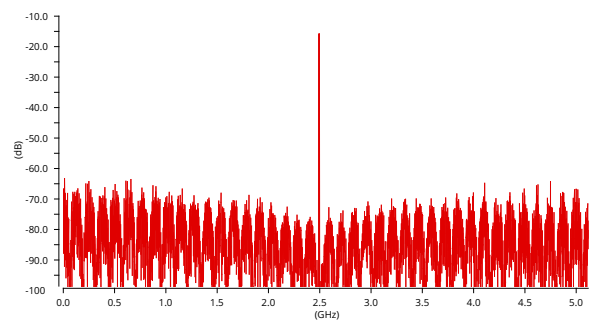

(b)

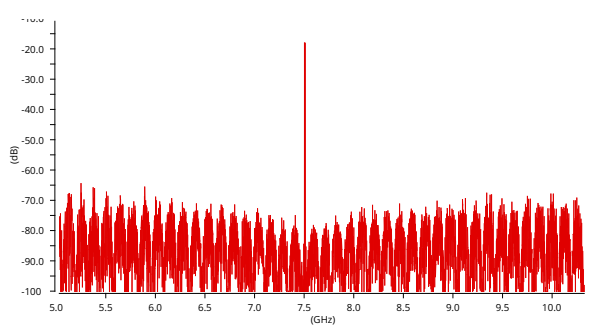

(d)

Figure 10: (a) Linearity at $2.5 \mathrm{GHz}$, (b) far out spectrum at $2.5 \mathrm{GHz}$, (c) linearity at $7.5 \mathrm{GHz}$, and (d) far out spectrum at $7.5 \mathrm{GHz}$.

the sampling frequency is at $600 \mathrm{MHz}$. No dynamic linearity performance is reported. In [10, a current steering mixing SDFIR RFDAC is reported that has an IM3 of $64.7 \mathrm{dBc}$ at $1 \mathrm{GHz}$ output frequency and the SDIFIR filter is only 6 taps. In [9, a 4th order FIR DAC is reported where each tap is a an 8-bit current steering DAC. The output frequency is at $900 \mathrm{MHz}$. In [17, a current steering SDFIR DAC is reported which achieves high dynamic range but operating at around $50 \mathrm{MHz}$ output frequency. All the above mentioned works are implemented in current steering structure and the linearity in high frequencies ( $\mathrm{GHz}$ operation) is limited mainly due to the limited output impedance of the unit current sources [10, 9, 17.

\section{Conclusion}

A direct digital-to-RF converter (DRFC) is designed and the analysis from system simulation and circuit-level simulation were presented in this work. Due 
to its digital-in-nature design, the DRFC benefits from technology scaling and can be monolithically integrated into advance digital VLSI systems. The 78order bandpass SDFIR filter is shown to be capable of removing the spectrallyshaped quantization noise of the fourth-order single-bit bandpass digital $\Sigma \Delta$ modulator. The simulation shows that the proposed DRFC which features a novel configurable voltage-mode RF DAC solution with a high linearity performance, can directly synthesize RF signals as high as $7.5 \mathrm{GHz}$, with excellent dynamic linearity performance.

\section{References}

\section{References}

[1] S. Balasubramanian, S. Boumaiza, H. Sarbishaei, T. Quach, P. Orlando, J. Volakis, G. Creech, J. Wilson, W. Khalil, Ultimate Transmission, IEEE Microwave Magazine 13 (1) (2012) 64-82. doi:10.1109/MMM. 2011. 2173983

[2] R. B. Staszewski, J. L. Wallberg, S. Rezeq, C.-M. Hung, O. E. Eliezer, S. K. Vemulapalli, C. Fernando, K. Maggio, R. Staszewski, N. Barton, M.-C. Lee, P. Cruise, M. Entezari, K. Muhammad, D. Leipold, All-digital PLL and transmitter for mobile phones, IEEE J. Solid-State Circuits 40 (12) (2005) 2469-2482. doi:10.1109/JSSC.2005.857417.

[3] D. C. Dinis, R. F. Cordeiro, F. M. Barradas, A. S. R. Oliveira, J. Vieira, Agile single- and dual-band all-digital transmitter based on a precompensated tunable delta sigma modulator, IEEE Trans. Microwave Theory Tech. 64 (12) (2016) 4720-4730. doi:10.1109/TMTT.2016.2622696

[4] E. Roverato, M. Kosunen, K. Cornelissens, S. Vatti, P. Stynen, K. Bertrand, T. Korhonen, H. Samsom, P. Vandenameele, J. Ryynanen, All-digital RF transmitter in 28nm CMOS with programmable RX-band noise shaping, in: Proc. IEEE Int. Solid-State Circuit Conf., 2017, pp. 222-223. doi: 10.1109/ISSCC.2017.7870341. 
[5] F. Yao, Q. Zhou, Z. Wei, A novel multilevel RF-PWM method with activeharmonic elimination for all-digital transmitters, IEEE Trans. Microwave Theory Tech. 66 (7) (2018) 3360-3373. doi:10.1109/TMTT.2018.2808946

[6] A. Jerng, C. Sodini, A wideband $\Sigma \Delta$ digital-RF modulator for high data rate transmitters, IEEE J. Solid-State Circuits 42 (8) (2007) 1710-1722. doi:10.1109/JSSC.2007.900255.

[7] M. M. Ebrahimi, M. Helaoui, F. M. Ghannouchi, Delta-sigma-based transmitters: Advantages and disadvantages, IEEE Microwave Magazine 14 (1) (2013) 68-78. doi:10.1109/MMM. 2012.2226541.

[8] J. J. McCue, B. Dupaix, L. Duncan, B. Mathieu, S. McDonnell, V. J. Patel, T. Quach, W. Khalil, A time-interleaved multimode $\Delta \Sigma$ RF-DAC for direct digital-to-RF synthesis, IEEE J. Solid-State Circuits 51 (5) (2016) 11091124. doi:10.1109/JSSC.2016.2521903.

[9] W. Gaber, P. Wambacq, J. Craninckx, M. Ingels, A CMOS IQ direct digital RF modulator with embedded RF FIR-based quantization noise filter, in:

(घ) Proc. Europ. Solid-State Circuits Conf., 2011, pp. 139-142. doi:10.1109/ ESSCIRC.2011.6044884.

${ }_{325}$ [10] S. M. Taleie, T. Copani, B. Bakkaloglu, S. Kiaei, A linear $\Sigma \Delta$ digital IF to RF DAC transmitter with embedded mixer, IEEE Trans. Microwave Theory Tech. 56 (5) (2008) 1059-1068. doi:10.1109/TMTT.2008.920156.

[11] A. Frappe, A. Flament, B. Stefanelli, A. Kaiser, A. Cathelin, An all-digital RF signal generator using high-speed delta sigma modulators, IEEE J.

330 ㄱolid-State Circuits 44 (10) (2009) 2722-2732. doi:10.1109/JSSC. 2009. 2028406

[12] F. T. Gebreyohannes, A. Frappe, A. Kaiser, A configurable transmitter architecture for IEEE 802.11ac and 802.11ad standards, IEEE Trans. Circuits Syst. II 63 (1) (2016) 9-13. doi:10.1109/TCSII.2015.2468920. 
[17] D. B. Barkin, A. C. Y. Lin, D. K. Su, B. A. Wooley, A CMOS oversampling bandpass cascaded D/A converter with digital FIR and current-mode semidigital filtering, IEEE J. Solid-State Circuits 39 (4) (2004) 585-593. doi: $10.1109 /$ JSSC. 2004.825245

[18] T. S. Doorn, E. Tuijl, D. Schinkel, A.-J. Annema, M. Berkhout, B. Nauta, An audio FIR-DAC in a BCD process for high power class-D amplifiers, in: 355

[19] M. T. Ozgun, M. Torlak, Effects of random delay errors in continuous-time semi-digital transversal filters, IEEE Trans. Circuits Syst. I 61 (1) (2014) 183-190. doi:10.1109/TCSI.2013.2264692.

[20] M. Sadeghifar, J. Wikner, O. Gustafsson, Linear programming design of 360 semi-digital FIR filter and $\Sigma \Delta$ modulator for VDSL2 transmitter, in: Proc. IEEE Int. Symp. Circuits Syst., 2014, pp. 2465-2468. doi:10.1109/ISCAS. 2014.6865672 
[21] H. Westerveld, D. Schinkel, E. van Tuijl, A 115dB-DR audio DAC with -61dBFS out-of-band noise, in: Proc. IEEE Int. Solid-State Circuit Conf., 2015, pp. 1-3. doi:10.1109/ISSCC.2015.7063033.

[22] M. R. Sadeghifar, O. Gustafsson, J. J. Wikner, Optimization problem formulation for semi-digital FIR digital-to-analog converter considering coefficients precision and analog metrics, Analog Integrated Circuits Signal Process doi: 10.1007/s10470-018-1370-7.

370 [23] S. Fukuda, S. Miya, M. Io, K. Hamashita, B. Nauta, Direct-digital modulation (DIDIMO) transmitter with $-156 \mathrm{dBc} / \mathrm{Hz} \mathrm{Rx}$-band noise using FIR structure, in: Proc. Europ. Solid-State Circuits Conf., 2012, pp. 53-56. doi:10.1109/ESSCIRC. 2012.6341254.

[24] A. Petraglia, S. Mitra, Effects of coefficient inaccuracy in switchedcapacitor transversal filters, IEEE Trans. Circuits Syst. 38 (9) (1991) 977983. doi:10.1109/31.83869

[25] A. Petraglia, Fundamental frequency response bounds of direct-form recursive switched-capacitor filters with capacitance mismatch, IEEE Trans. Circuits Syst. II 48 (4) (2001) 340-350. doi:10.1109/82.933792.

[26] S. Luschas, R. Schreier, H.-S. Lee, Radio frequency digital-to-analog converter, IEEE J. Solid-State Circuits 39 (9) (2004) 1462-1467. doi:10. 1109/JSSC. 2004.829377.

[27] B. Farley, C. Erdmann, B. Vaz, J. McGrath, E. Cullen, B. Verbruggen, R. Pelliconi, D. Breathnach, P. Lim, A. Boumaalif, P. Lynch, C. Mesadri, ${ }_{385}$ D. Melinn, K. P. Yap, L. Madden, A programmable RFSoC in 16nm FinFET technology for wideband communications, in: Proc. IEEE Asian Solid State Circuits Conf., 2017, pp. 1-4. doi:10.1109/ASSCC. 2017.8240201.

[28] M. Sadeghifar, J. Wikner, A higher Nyquist-range DAC employing sinua soidal interpolation, in: NORCHIP, 2010, pp. 1-4. doi:10.1109/NORCHIP. 2010.5669460 
[29] C. H. Lin, F. M. L. van der Goes, J. R. Westra, J. Mulder, Y. Lin, E. Arslan, E. Ayranci, X. Liu, K. Bult, A 12 bit 2.9 GS/s DAC with IM3<60 dBc beyond $1 \mathrm{GHz}$ in $65 \mathrm{~nm}$ CMOS, IEEE J. Solid-State Circuits 44 (12) (2009) 3285-3293. doi:10.1109/JSSC.2009.2032624.

[35] P. R. Kinget, Device mismatch and tradeoffs in the design of analog circuits,

[36] F. T. Gebreyohannes, A. Frappe, P. Cathelin, A. Cathelin, A. Kaiser, All-

प

[30] P. Palmers, M. S. J. Steyaert, A 10 bit 1.6-GS/s 27-mW current-steering D/A converter with 550-MHz 54-dB SFDR bandwidth in 130-nm CMOS,

【 IEEE Trans. Circuits Syst. I 57 (11) (2010) 2870-2879. doi:10.1109/ TCSI.2010.2052491.

[31] R. Schreier, G. C. Temes, Understanding Delta-Sigma Data Converters, Wiley-IEEE Press, 2004.

[32] C. H. Lin, K. L. J. Wong, T. Y. Kim, G. R. Xie, D. Major, G. Unruh, S. R. Dommaraju, H. Eberhart, A. Venes, A 16b 6GS/S nyquist DAC with imd $<-90 \mathrm{dBc}$ up to $1.9 \mathrm{GHz}$ in $16 \mathrm{~nm}$ CMOS, in: Proc. IEEE Int. Solid-State Circuit Conf., 2018, pp. 360-362. doi:10.1109/ISSCC.2018.8310333.

[33] A. Gersho, B. Gopinath, A. M. Odlyzko, Coefficient inaccuracy in transversal filtering, The Bell System Technical Journal 58 (10) (1979) 2301-2316. doi:10.1002/j.1538-7305.1979.tb02968.x.

[34] M. J. M. Pelgrom, A. C. J. Duinmaijer, A. P. G. Welbers, Matching properties of MOS transistors, IEEE J. Solid-State Circuits 24 (5) (1989) 14331439. doi:10.1109/JSSC.1989.572629.

IEEE J. Solid-State Circuits 40 (6) (2005) 1212-1224. doi:10.1109/JSSC. 2005.848021 digital transmitter architecture based on two-path parallel 1-bit high pass filtering dacs, IEEE Trans. Circuits Syst. I 65 (11) (2018) 3956-3969. doi: 10.1109/TCSI.2018.2853992 